\title{
Gonadotropin-Releasing Hormone Agonist Based Fertility-Sparing Treatment in Women with Endometrial Carcinoma or Atypical Endometrial Hyperplasia
}

\author{
chen junyu ${ }^{1}$, Dongyan $\mathrm{Cao}^{2}$, jiaxin yang ${ }^{1}$, mei $\mathrm{yu}^{1}$, huimei $\mathrm{zhou}^{1}$, ninghai cheng ${ }^{1}$, jinhui \\ wang $^{1}$, ying zhang ${ }^{1}$, peng peng ${ }^{1}$, and keng shen ${ }^{1}$ \\ ${ }^{1}$ Affiliation not available \\ ${ }^{2}$ Peking Union Medical College Hospital
}

September 30, 2021

\begin{abstract}
Objectives:To evaluate the efficacy and safety of gonadotropin-releasing hormone agonist (GnRHa) combined with levonorgestrelreleasing intrauterine system (LNG-IUS) or aromatase inhibitor (AI) in women with endometrial carcinoma (EC) and atypical endometrial hyperplasia (AEH) who wish to preserve their fertility. Design: A single-center restrospective study. Setting: Department of Obstetrics and Gynecology, Peking Union Medical College Hospital Population:179 patients with early stage EC or AEH who wish to preserve their fertility. Methods: Patients were treated with the combination of GnRHa with LNG-IUS (group GLI: GnRHa IH every 4 weeks and LNG-IUS insertion constantly) or combination of GnRHa with AI (group GAI: GnRHa IH every 4 weeks and oral letrozole $2.5 \mathrm{mg}$, daily). Histological evaluation was performed at the end of each course (every $3-4$ months) by hysteroscopy and curettage. All patients were followed up regularly. Main outcome measures: Pathological response to treatmen, categorized as complete response (CR), partial response (PR), stable disease (SD), and progressive disease (PD). Results: Overall, 169 (94.4\%) patients achieved CR, 96.7\% in AEH and 93.3\% in EC patients. The median time to CR was 6 (3-18) months, 4 (3-10) months in AEH and 8 (3-18) months in EC patients. After a median follow up of 27.5 months, 41 (24.3\%) women developed recurrence with the median recurrence time of 17 (6-77) months. Of the patients with CR, 134 cases desired to conceive, and $42(32.3 \%)$ patients became pregnant. Conclusion: GnRHa based fertility-sparing treatment achieved good treatment outcomes. Future larger multi-institutional studies should be designed to confirm these preliminary findings.
\end{abstract}

\section{Introduction}

Endometrial cancer (EC) is one of the most common as well as an increasingly problematic gynecological cancer, whose incidence has gradually risen in recent years ${ }^{(1-3)}$. EC and its precursor, atypical endometrial hyperplasia (AEH), affect a larger proportion of premenopausal women, including many women of childbearing age ${ }^{(4)}$. The standard treatment for this disease requires a hysterectomy with bilateral salpingooophorectomy, with or without pelvic sentinel lymph node dissection, pelvic or para-aortic lymphadenectomy. However, this standard treatment results in a permanent loss of fertility while young patients have a strong desire to bear children. Therefore, conservative treatment should be discussed in young patients with earlystage $\mathrm{EC}$ or $\mathrm{AEH}$ who wish to preserve their fertility.

High-dose progestins including medroxyprogesterone acetate (MPA) and megestrol acetate (MA) have been the mainstay of conservative treatment of AEH and early-stage $\mathrm{EC}^{(5-7)}$. But adverse effects such as weight gain, liver dysfunction and resistance to oral progestins lead to seeking alternative treatments is mandatory. At present, gonadotropin-releasing hormone agonist (GnRHa) plus levonorgestrel-releasing intrauterine system (LNG-IUS) could be used as an alternative to oral-systemic progestin for the treatment of women with $\mathrm{EC}$ and $\mathrm{AEH}^{(8)}$. Besides, combination of GnRHa and Aromatase inhibitors (AIs) has also been reported as 
an option for preserve women's fertility with EC and proved to have a good response in previous study ${ }^{(9)}$. But the data on effective application of GnRHa based combination treatment were relatively limited. Therefore, further studies should be performed.

The aim of this study was to investigate the efficacy and safety of the combination of GnRHa plus LNG-IUS or AI (letrozole) in young women with EC or AEH.

\section{Methods}

\subsection{Patients recruited}

All patients who received GnRHa based therapy between January 2013 and December 2020. Patients' information was collected from medical records and a prospectively maintained database, which represented a standard protocol was followed for all patients. The inclusion criteria were as follows after systematic pre-treatment evaluation : (1) Women of 18-45 years old who desire to preserve their fertility; (2) Histologically confirmed AEH or Grade 1-2 endometrioid adenocarcinoma; (3) No signs of myometrial invasion or extra-uterine metastasis by enhanced magnetic resonance imaging (MRI); (4) who were contra-indicated or unsuitable for oral progestin; (5) Written informed consent obtained; (6) Patients were followed up regularly, full text and complete data available. This study was approved by the Ethics Committee of our hospital

\subsection{Treatments}

Two regimens were used; (1) Regime GLI: combination of subcutaneous injection of $3.75 \mathrm{mg}$ GnRHa every 4 weeks and LNG-IUS (Mirena; Bayer Health Care Pharmaceutical Inc) insertion constantly; (2) Regime GAI: combination of subcutaneous injection of $3.75 \mathrm{mg}$ GnRHa every 4 weeks and oral AI (letrozole) $2.5 \mathrm{mg}$ daily.

During the process of treatment, weight loss plans including diet control and exercise recommendation were provided to all patients. Outpatient visits were arranged during the treatment, symptoms such as vaginal spotting, abdominal pain were recorded, physical examination including body weight and lab tests including complete blood counts and biochemistry panels were performed. Trans-vaginal ultrasound scan was performed at every visit to assess the endometrium. Histological response was determined by endometrial curettage under hysteroscopic evaluation every 3-4 months (one course) during the treatment.

\subsection{Response Evaluation}

Pathological response to treatment was categorized as complete response $(\mathrm{CR})$, partial response $(\mathrm{PR})$, stable disease (SD), and progressive disease (PD). CR was defined as the absence of evidence of hyperplasia or carcinoma. PR was defined as histological regression or endometrial decidual change. SD was defined as the persistence of disease as initially diagnosed. PD was defined as progression to a lesion of higher grade or progressive disease including myometrial invasion, extra-uterine disease, or lymph node metastasis ${ }^{(10)}$. Those who had not achieve CR after 12 months of therapy were considered to have failed fertility-preserving treatment and were recommend to receive surgery. Once achieved CR, patients who desire to get pregnant were encouraged to conceive or referred to underwent assisted reproductive technology (ART). Those with CR who had no birth plans were prescribed oral contraceptives cyclic progestin or LNG-IUS insertion to prevent recurrence.

\subsection{Follow up}

After the documentation of CR, all patients were regularly followed up for a prolonged period with 3-6month intervals. During each follow-up visit, the following information was collected: menstruation period or abnormal vaginal bleeding, results of trans-vaginal ultrasound scan or MRI if necessary, data relating relapse (interval between $\mathrm{CR}$ and recurrence, diagnosis of recurrence, treatment and survival outcomes). Fertility outcomes including time of gestation, using of ART, obstetrical complications and delivery were also documented. If the patient received hysterectomy, the reason and histological results of the surgery were also collected. 


\subsection{Statistical analyses}

Statistical analysis was performed using IBM SPSS for Windows (version 22.0). Categorical variables are summarized in frequency tables, whereas continuous variables are presented as median (range). Frequency distributions were compared using chi-squared or Fisher's exact tests and median values were compared using Mann-Whitney U tests. Logistic regression models were built to determine associations of CR, recurrence and fertility. For all statistical tests, the differences were considered statistically significant when P-values were $<0.05$.

\section{Results}

\subsection{Demographics and Clinical manifestations}

The clinicopathologic characteristics of the patients are summarized in Table 1. Sixty (33.5\%) patients were diagnosed as AEH and $119(66.5 \%)$ were diagnosed as EC. The median age at diagnosis was 31 years, ranging from 21 to 43 years. 163 (91.1\%) women were nulliparous, $48(26.8 \%)$ had comorbidity including polycystic ovary syndrome (PCOS) and/or endometriosis and $72(40.2 \%)$ patients were obese.

\subsection{Treatment Effects}

Totally, 169 (94.4\%) achieved CR with the 6 months (3-18 months) median CR time (Table2). 10 (5.59\%) patients failed to CR, $4 \mathrm{PR}$ and $6 \mathrm{SD}$, then underwent the hysterectomy with or without lymphadenectomy. According to the postoperative pathological diagnosis, 2 of them were diagnosed as AEH, 7 were diagnosed as stage IA, and one combined with stage IC ovarian endometrial carcinoma. All patients alive without tumor at the final contact.

The CR rate was $96.7 \%$ in AEH patients and $93.3 \%$ in EC patients $(\mathrm{p}=0.351$, ) and the median time to CR was 4 months (3-10 months) in AEH patients and 8 months (3-18 months) in EC patients, respectively. At the end of the first therapy course, the CR rate in AEH was higher than EC (65\% vs $31.9 \%, \mathrm{p}<0.0001$ ). Similar results were also found in the second course (91.7\% vs $72.3 \%, \mathrm{p}=0.003)$. The CR rate in obese and non-obese patients were $95.3 \%$ and $93.1 \%$ ( $\mathrm{p}=0.516)$, respectively. Patients with weight loss more than $3 \%$ of their initial body weight had a higher response rate $(98.8 \%$ vs $90.9 \%, \mathrm{p}=0.023)$.

\subsection{Adverse Effects}

Postmenopausal symptoms such as hot flashes and vaginal dryness was the most common adverse effect (17.3\%), followed by irregular bleeding (11.2\%) and abnormal liver function (1.7\%). The degree of menopause symptoms was minor and no patients received add-back estrogen. IUD dislocation occurred in 2 patients and was resolved by reinsertion of the IUD. The scheduled treatment was not delayed due to these side effects. No treatment-related deaths were identified.

\subsection{Follow up}

After pathologically CR achieved, 147 patients accepted maintenance treatment including LNG-IUS, cyclical oral contraceptives or low-dose cyclic progestin until they began attempting gestation. Other 22 patients did not receive any treatment, just follow up regularly. After a median follow-up time of 27.5 months (3-92 months), 41 (24.3\%) women had developed recurrence. The median time to recurrence was 17 months, ranging from 6-77 months. 10 patients who gave up to preserve their uterus chose to receive hysterectomy with or without lymphadenectomy. Extrauterine lesions were identified in 2 patients and received adjuvant therapy after surgery.

31 patients received fertility sparing re-treatment after recurrence, and $26(83.9 \%)$ achieved CR again. Hysterectomy was performed in $3(9.7 \%)$ patients due to SD or PD. The rest of 3 patients were still in treatment at the final contact. No patient died of the disease during this period.

The related factors for recurrence were shown in Table 3. Multivariate analysis indicated that the recurrence rate was higher in patients who over 30 years $(32.3 \%$ vs $13.8 \%, \mathrm{p}=0.010)$, and who lost lower than $3 \%$ of 
their weight ( $32.2 \%$ vs $15.1 \%, \mathrm{p}=0.022)$. The disease-free survival (DFS) of patients were shown in Figure 1.

\subsection{Fertility Outcomes}

After achieving CR, 134 women attempted to get pregnant and 75 (56.0\%) women were transferred to receive ART. Totally, $42(32.3 \%)$ patients became pregnant, 24 (17.9\%) of them successfully delivered and $5(3.7 \%)$ were in pregnancy, while 13 of them miscarried, 9 at the first trimester and 4 at the second trimester. The median duration from CR to pregnancy was 12 months (1-72 months). In univariate analysis, pregnancy rate was superior in patients who were younger than 35 years old $(37.6 \%$ vs. $12.1 \%, \mathrm{p}=0.06)$. Higher probability was observed in non-obese patients $(39.0 \%$ vs $19.23 \%, \mathrm{p}=0.016)$. ART showed high tendency of pregnancy ( $38.7 \%$ vs $13.7 \%, \mathrm{p}=0.066)$, and high pregnancy rate was observed in IVF-ET $(47.8 \%$ vs $27.9 \%, \mathrm{p}=0.039)$. Age and obesity remained significant when multivariate regression analysis was applied (Table 4).

\section{Discussion}

With increasing incidence of EC in younger women, increasingly women are likely to seek conservative management options. Progestin therapy is widely accepted as the main fertility-sparing treatment for young women with $\mathrm{AEH}$ and well-differentiated $\mathrm{EC}$ and acquire satisfactory results ${ }^{(5-7)}$. However, there are still about $20 \%$ of these patients who failed to achieve CR, and lost fertility after hysterectomy ${ }^{(11)}$. Side-effects or contraindications of progestins, such as weight gain or liver dysfunction call for alternative regimes other than oral high dose progestins. ${ }^{(12)}$.

GnRHa is a group of synthetic compounds which are derived from natural GnRH through substitution of amino acids at position 6 and/or $10^{(13)}$. Some studies revealed that GnRHa can affect endometrial cell proliferation not only through the hormonal axis indirectly, but also by acting on the GnRH receptors directly ${ }^{(14,15)}$. Therefore, GnRHa could be used in the treatment of endometrial diseases due to its antiproliferative effect on endometrial cells. The application of GnRHa for endometrial diseases was initially reported for the management of patients with recurrent EC, resistant to other treatment modalities, with a regression rate as high as $35 \%(16,17)$. LNG-IUS represents a newly available delivery system for EC treatment ${ }^{(18)}$. It could provide local intrauterine concentrations many-fold higher than oral progestins. Some researchers used LNG-IUS solely or in combination with GnRHa and reported encouraging results ${ }^{(9,19,20)}$. Letrozole is the third-generation AIs, which can reduce the levels of estrogen by inhibiting estrogen synthesis leading to a reduction in the receptor-mediated growth stimulated in estrogen receptor positive tumors such as EC. Combination of GnRHa and AIs has been reported as an option for preserve women's fertility with EC and $\mathrm{AEH}^{(21,22)}$. In this study, we reported a series of patients with EC and AEH treated with GnRHa plus LNG-IUS/AIs. The preliminary results show an encouraging result a little better than or at least comparable to previous oral-progestin studies $(23,24)$.

\section{Main findings}

In our study, over 90\% patients achieved CR, 96.7\% in AEH patients and 93.3\% in EC patients, suggesting an encouraging result. Most AEH patients achieved CR within 6 months, but the median CR time of EC is about 3-4 months longer than AEH. 18 patients achieved CR after extension of treatment time as long as 9-15 months. Thus, we recommend that these combination regimes be administered for at least 6 months, especially for EC patients. The long-term adverse effect of GnRHa and influence on fertility by repeated curettage need to be noted. It has been documented that $2-3 \%$ of bone mass will be loss with 6 months use of GnRH analogs. And it is unclear what's the maximal duration of therapy, whether the add-back therapy should be performed, whether the bone mineral density should be monitored, and whether calcium and bisphosphonates should be added ${ }^{(25)}$. Condsidering that over $90 \%$ AEH patients acquired complete remission after 2 course of treatments and LNG-IUS could be used solely to treat $\mathrm{EC}^{(18.19)}$, for AEH patients who already achieved PR after 6 months, the use of LNG-IUD alone might be an option to avoid the side effect of GnRHa. But the efficacy and safety were unclear and further research is needed to accumulate experience. 
In our study, patients who were obese and lose weight $<3 \%$ have lower response and pregnancy rates, as well as higher recurrence rates, consistent with previous studies ${ }^{(26)}$. Patients were unable to conceive due to obesity and PCOS, which leading to anovulation and the absence of stimulation of progestin, may also increase the risk of recurrence ${ }^{(27)}$. Herein, weight control and health consulting are crucial in the wholelifespan management of fertility-spearing treatment. GnRHa combined therapy have advantage on weight control compared with progestin therapy since we all know that weight gain was a main side effect of high-dose progestin.

Due to the data limitation, we failed to find the biomarker and the possible reason for treatment failure. MMR testing has been proposed in young women desiring fertility-sparing treatment, but the association between MMR and response is unclear ${ }^{(28)}$. Some articles have proposed that the overall and recurrence-free survival was significantly lower in p53 abnormal and dMMR patient subgroups. Thus, patients with Lynch syndrome and P53 mutations may not be treated conservatively ${ }^{(29)}$. The status of progesterone receptor (PR) and estrogens receptor (ER) was thought to be associated with disease regression in some research ${ }^{(30-32)}$. But all patients in our study were ER and PR positive, so, we failed to analyse the relationship between IHC and of CR. As modern The Cancer Genome Atlas (TCGA)-based molecular classification system that has been validated, it might helpful to predict the response and contribute to the selection of population who suit for fertility-preserving.

Previous studies revealed a high rate of relapse, ranging from $10 \%$ to $88 \%(18,24)$. In our research, about $25 \%$ women had developed recurrence with 20 months median recurrence time, consist with former articles. But some of the recurrences were diagnosed as early as 6-7 months after a CR. Another study report that recurrence occurred 3-4 months after CR which mandates the follow-up to be started early ${ }^{(33)}$. The latest recurrence in our cohort took place at 7 years in our research, others also report at 13 years ${ }^{(34,35)}$. Therefore, long-term and regular follow-up is essential due to the high rate of late recurrence. Additionally, hormonal maintenance therapy is important for complete responders who do not wish to conceive immediately after completion of treatment ${ }^{(36)}$. And low recurrence rate was also found in patients with pregnancy. Therefore, maintenance therapy and conception immediately were encouraged to reduce the risk of recurrence.

The pregnancy and live birth rates in our research are still somewhat suboptimal, lower than other large studies about progestin $^{(23,37)}$. But the miscarriage rate at the first or second trimester are in accordance with ordinary population ${ }^{(38)}$. This might be due to endometrium atrophic decreased by repeated hysteroscopic evaluation and curettage. Moreover, the follow up time in our study was relatively short, if longer follow-up time were performed, high rate of relapse and live birth may be observed ${ }^{(39)}$. Despite our expectations ART did not significantly improve the live birth rate, women who choosing IVF-ET had a relatively better results and some studies did report improved birth rate with $\mathrm{ART}^{(40,41)}$. Hence, once CR has been achieved, pregnancy should be carried out as soon as possible, and IVF-ET is recommended without causing significant delays.

\section{Strengths and limitations}

To the best of our knowledge, the current study included the largest number of subjects of both oncologic and reproductive results about GnRHa based treatments. The findings confirm that the combination of GnRHa with LNG-IUS/letrozole is an effective method with high rate of regression and minor side effects. But there are still some limitations. Firstly, it is a single-center retrospective study, multi-center prospective clinical trials are supposed to conducted to verify the suitability of the combination of GnRHa with LNGIUS/letrozole use for fertility preservation. Secondly, the follow-up time of this center is limited, and longterm follow-up of these patients will also be performed to verify high pregnancy rate. Thirdly, as the modern TCGA-based molecular classification system is being introduced, it would be necessary to assess responses and recurrences in all future conservatively treated EC patients from this molecular perspective. Fourthly, long-term side-effect of GnRHa such as osteoporosis and the cardiovascular complications should be considered in future observation.

\section{Conclusion}


In conclusion, the findings of our study confirm that the combination of GnRHa with LNG-IUS/letrozole is an effective method with high rate of regression. Besides, the recurrence and pregnancy rate are comparable to traditional oral-progestin therapy. So, the combination of GnRHa with LNG-IUS/letrozole is an encouraging alternative regime for fertility-preserving of EC and AEH patients.

\section{Author contributions}

JC, JY, DC, and KS: conceived and designed the study. JC, MY, HZ, JW, YZ, NC, and PP: data acquisition. JC, and DC: analyzed the data. JC: wrote the original draft. JY, DC, and KS: wrote, reviewed, and edit. All authors contributed to the article and approved the submitted version.

\section{Acknowledgements}

The authors thank all of the faculty, nurses, and staff at Department of Obstetrics \& Gynecology in PUMCH for the excellent care they provide for patients. The authors also sincerely thank all the patients and their family members for their contribution to this research effort.

\section{Funding:}

National Natural Science Foundation of China (81101975)

\section{Ethical statement}

This study was approved by the Ethics Committee of PUMCH in 07-07-2009 (S-244)

References

1. Lu KH, Broaddus RR. Endometrial Cancer. N Engl J Med. 2020;383(21):2053-64.

2. Zhang S, Gong TT, Liu FH, et al. Global, Regional, and National Burden of Endometrial Cancer, 1990-2017: Results From the Global Burden of Disease Study, 2017. Front Oncol. 2019;9:1440.

3. Siegel RL, Miller KD, Jemal A. Cancer statistics, 2019. CA Cancer J Clin. 2019;69(1):7-34.

4. Li M, Guo T, Cui R, et al. Weight control is vital for patients with early-stage endometrial cancer or complex atypical hyperplasia who have received progestin therapy to spare fertility: a systematic review and meta-analysis. Cancer Manag Res. 2019;11:4005-21.

5. Gunderson CC, Fader AN, Carson KA, et al. Oncologic and reproductive outcomes with progestin therapy in women with endometrial hyperplasia and grade 1 adenocarcinoma: a systematic review. Gynecol Oncol. $2012 ; 125(2): 477-82$.

6. Guillon S, Popescu N, Phelippeau J, et al. A systematic review and meta-analysis of prognostic factors for remission in fertility-sparing management of endometrial atypical hyperplasia and adenocarcinoma. Int J Gynaecol Obstet. 2019;146(3):277-88.

7. Yu M, Yang JX, Wu M, et al. Fertility-preserving treatment in young women with well-differentiated endometrial carcinoma and severe atypical hyperplasia of endometrium. Fertil Steril. 2009;92(6):2122-4.

8. Pashov AI, Tskhay VB, Ionouchene SV. The combined GnRH-agonist and intrauterine levonorgestrelreleasing system treatment of complicated atypical hyperplasia and endometrial cancer: a pilot study. Gynecol Endocrinol. 2012;28(7):559-61.

9. Zhou H, Cao D, Yang J, et al. Gonadotropin-Releasing Hormone Agonist Combined With a Levonorgestrel-Releasing Intrauterine System or Letrozole for Fertility-Preserving Treatment of Endometrial Carcinoma and Complex Atypical Hyperplasia in Young Women. Int J Gynecol Cancer. 2017;27(6):1178-82.

10. Yu M, Wang Y, Yuan Z, et al. Fertility-Sparing Treatment in Young Patients With Grade 2 Presumed Stage IA Endometrioid Endometrial Adenocarcinoma. Front Oncol. 2020;10:1437. 
11. Gallos ID, Yap J, Rajkhowa M, et al. Regression, relapse, and live birth rates with fertility-sparing therapy for endometrial cancer and atypical complex endometrial hyperplasia: a systematic review and metaanalysis. Am J Obstet Gynecol. 2012;207(4):266.e1-12.

12. Knez J, Al Mahdawi L, Takač I, et al. The Perspectives of Fertility Preservation in Women with Endometrial Cancer. Cancers (Basel). 2021;13(4).

13. Grimbizis G, Tsalikis T, Tzioufa V, et al. Regression of endometrial hyperplasia after treatment with the gonadotrophin-releasing hormone analogue triptorelin: a prospective study. Hum Reprod. 1999;14(2):47984.

14. Gallos ID, Shehmar M, Thangaratinam S, et al. Oral progestogens vs levonorgestrel-releasing intrauterine system for endometrial hyperplasia: a systematic review and metaanalysis. Am J Obstet Gynecol. 2010;203(6):547.e1-10.

15. Jeyarajah AR, Gallagher CJ, Blake PR, et al. Long-term follow-up of gonadotrophin-releasing hormone analog treatment for recurrent endometrial cancer. Gynecol Oncol. 1996;63(1):47-52.

16. Gallagher CJ, Oliver RT, Oram DH, et al. A new treatment for endometrial cancer with gonadotrophin releasing-hormone analogue. Br J Obstet Gynaecol. 1991;98(10):1037-41.

17. De Vriese G, Bonte J. Possible role of goserelin, an LH-RH agonist in the treatment of gynaecological cancers. Eur J Gynaecol Oncol. 1993;14(3):187-91.

18. Leone Roberti Maggiore U, Martinelli F, Dondi G, et al. Efficacy and fertility outcomes of levonorgestrelreleasing intra-uterine system treatment for patients with atypical complex hyperplasia or endometrial cancer: a retrospective study. J Gynecol Oncol. 2019;30(4):e57.

19. Pal N, Broaddus RR, Urbauer DL, et al. Treatment of Low-Risk Endometrial Cancer and Complex Atypical Hyperplasia With the Levonorgestrel-Releasing Intrauterine Device. Obstet Gynecol. 2018;131(1):109-16.

20. Minig L, Franchi D, Boveri S, et al. Progestin intrauterine device and GnRH analogue for uterus-sparing treatment of endometrial precancers and well-differentiated early endometrial carcinoma in young women. Ann Oncol. 2011;22(3):643-9.

21. Barker LC, Brand IR, Crawford SM. Sustained effect of the aromatase inhibitors anastrozole and letrozole on endometrial thickness in patients with endometrial hyperplasia and endometrial carcinoma. Curr Med Res Opin. 2009;25(5):1105-9.

22. Azim A, Oktay K. Letrozole for ovulation induction and fertility preservation by embryo cryopreservation in young women with endometrial carcinoma. Fertil Steril. 2007;88(3):657-64.

23. Chen M, Jin Y, Li Y, et al. Oncologic and reproductive outcomes after fertility-sparing management with oral progestin for women with complex endometrial hyperplasia and endometrial cancer. Int J Gynaecol Obstet. 2016;132(1):34-8.

24. Yamagami W, Susumu N, Makabe T, et al. Is repeated high-dose medroxyprogesterone acetate (MPA) therapy permissible for patients with early stage endometrial cancer or atypical endometrial hyperplasia who desire preserving fertility? J Gynecol Oncol. 2018;29(2):e21.

25. Yang BY, Gulinazi Y, Du Y, et al. Metformin plus megestrol acetate compared with megestrol acetate alone as fertility-sparing treatment in patients with atypical endometrial hyperplasia and well-differentiated endometrial cancer: a randomised controlled trial. Bjog. 2020;127(7):848-57.

26. Shafiee MN, Chapman C, Barrett D, et al. Reviewing the molecular mechanisms which increase endometrial cancer (EC) risk in women with polycystic ovarian syndrome (PCOS): time for paradigm shift? Gynecol Oncol. 2013;131(2):489-92. 
27. Luo J, Chlebowski RT, Hendryx M, et al. Intentional Weight Loss and Endometrial Cancer Risk. J Clin Oncol. 2017;35(11):1189-93.

28. Soslow RA, Tornos C, Park KJ, et al. Endometrial Carcinoma Diagnosis: Use of FIGO Grading and Genomic Subcategories in Clinical Practice: Recommendations of the International Society of Gynecological Pathologists. Int J Gynecol Pathol. 2019;38 Suppl 1(Iss 1 Suppl 1):S64-s74.

29. Britton H, Huang L, Lum A, et al. Molecular classification defines outcomes and opportunities in young women with endometrial carcinoma. Gynecol Oncol. 2019;153(3):487-95.

30. Raffone A, Travaglino A, Zullo FM, et al. Predictive Accuracy of Progesterone Receptor B in Young Women with Atypical Endometrial Hyperplasia and Early Endometrial Cancer Treated with Hysteroscopic Resection plus LNG-IUD Insertion. J Minim Invasive Gynecol. 2021;28(6):1244-53.

31. Raffone A, Travaglino A, Saccone G, et al. Should progesterone and estrogen receptors be assessed for predicting the response to conservative treatment of endometrial hyperplasia and cancer? A systematic review and meta-analysis. Acta Obstet Gynecol Scand. 2019;98(8):976-87.

32. Travaglino A, Raffone A, Saccone G, et al. Immunohistochemical predictive markers of response to conservative treatment of endometrial hyperplasia and early endometrial cancer: A systematic review. Acta Obstet Gynecol Scand. 2019;98(9):1086-99.

33. Novikova OV, Nosov VB, Panov VA, et al. Live births and maintenance with levonorgestrel IUD improve disease-free survival after fertility-sparing treatment of atypical hyperplasia and early endometrial cancer. Gynecol Oncol. 2021.

34. Fujiwara H, Jobo T, Takei Y, et al. Fertility-sparing treatment using medroxyprogesterone acetate for endometrial carcinoma. Oncol Lett. 2012;3(5):1002-6.

35. Wang CJ, Chao A, Yang LY, et al. Fertility-preserving treatment in young women with endometrial adenocarcinoma: a long-term cohort study. Int J Gynecol Cancer. 2014;24(4):718-28.

36. Wang $\mathrm{Y}$, Zhou R, Wang H, et al. Impact of treatment duration in fertility-preserving management of endometrial cancer or atypical endometrial hyperplasia. Int J Gynecol Cancer. 2019;29(4):699-704.

37. Chae SH, Shim SH, Lee SJ, et al. Pregnancy and oncologic outcomes after fertility-sparing management for early stage endometrioid endometrial cancer. Int J Gynecol Cancer. 2019;29(1):77-85.

38. Ammon Avalos L, Galindo C, Li DK. A systematic review to calculate background miscarriage rates using life table analysis. Birth Defects Res A Clin Mol Teratol. 2012;94(6):417-23.

39. Gallos ID, Gupta JK. Comment on: What about the relapse of endometrial hyperplasia? Eur J Obstet Gynecol Reprod Biol. 2013;171(2):e5.

40. Hertz-Picciotto I, Samuels SJ. Incidence of early loss of pregnancy. N Engl J Med. 1988;319(22):1483-4.

41. Park JY, Seong SJ, Kim TJ, et al. Pregnancy outcomes after fertility-sparing management in young women with early endometrial cancer. Obstet Gynecol. 2013;121(1):136-42.

Table 1 Clinicopathologic characteristics of the patients

\begin{tabular}{ll}
\hline Characteristics & Total $\mathrm{n}(\%)$ \\
$\mathrm{n}$ & 179 \\
$\mathrm{BMI}, \mathrm{kg} / \mathrm{m} 2$ (median,range) & $29.2(17.7-46.1)$ \\
Obesity & $72(40.2 \%)$ \\
Age, years(median,range) & $31(21-43)$ \\
Comorbidity & $40(22.3 \%)$ \\
PCOS & $9(5.0 \%)$ \\
Endometriosis &
\end{tabular}




\begin{tabular}{ll} 
Histology & \\
AEH & $60(33.5 \%)$ \\
EC & $119(66.5 \%)$ \\
\hline
\end{tabular}

Notes: $\mathrm{BMI}=$ body mass index, $\mathrm{PCOS}=$ polycystic ovary syndrome, $\mathrm{AEH}=$ atypical endometrial hyperplasia, $\mathrm{EC}=$ endometrial carcinoma.

Table 2 Duration of complete remission

\begin{tabular}{lllll}
\hline Times & AEH & EC & Total & p-value \\
1 course & $65 \%(39)$ & $31.9 \%(38)$ & $43.0 \%(77)$ & $<\mathbf{0 . 0 0 0 1}$ \\
2 courses & $91.7 \%(55)$ & $72.3 \%(86)$ & $78.8 \%(141)$ & $\mathbf{0 . 0 0 3}$ \\
3 courses & $96.7 \%(58)$ & $89.1 \%(106)$ & $91.6 \%(164)$ & 0.145 \\
Total & $96.7 \%(58)$ & $93.3 \%(111)$ & $94.4 \%(169)$ & 0.351 \\
\hline
\end{tabular}

Notes: $\mathrm{AEH}=$ atypical endometrial hyperplasia, $\mathrm{EC}=$ endometrial carcinoma.

Table 3 Risk factors of recurrence

\begin{tabular}{|c|c|c|c|c|}
\hline $\begin{array}{l}\text { Risk factors to } \\
\text { recurrence }\end{array}$ & $\begin{array}{l}\text { Univariate analysis } \\
\text { HR }(95 \% \text { CI })\end{array}$ & P-value & $\begin{array}{l}\text { Multivariate } \\
\text { analysis HR (95\% } \\
\text { CI) }\end{array}$ & $\mathrm{P}$-value \\
\hline $\begin{array}{l}\text { Age: [?]30 years } \\
\text { vs }<30 \text { years }\end{array}$ & $\begin{array}{l}2.903 \\
(1.340-6.289)\end{array}$ & 0.006 & $\begin{array}{l}2.935 \\
(1.298-6.639)\end{array}$ & 0.010 \\
\hline AEH vs EC & $\begin{array}{l}0.438 \\
(0.157-0.223)\end{array}$ & 0.155 & & \\
\hline $\begin{array}{l}\text { Comorbidity: Yes } \\
\text { vs No }\end{array}$ & $\begin{array}{l}1.556 \\
(0.728-3.324)\end{array}$ & 0.010 & $\begin{array}{l}2.098 \\
(0.908-4.848)\end{array}$ & 0.082 \\
\hline $\begin{array}{l}\text { Obesity: Yes vs } \\
\text { No }\end{array}$ & $\begin{array}{l}1.758 \\
(0.848-3.756)\end{array}$ & 0.127 & & \\
\hline $\begin{array}{l}\text { Loss-weight: }<3 \% \\
\text { vs [?] } 3 \%\end{array}$ & $\begin{array}{l}2.654 \\
(1.234-5.658)\end{array}$ & 0.010 & $\begin{array}{l}2.719 \\
(1.159-6.380)\end{array}$ & 0.022 \\
\hline $\begin{array}{l}\text { Maintenance } \\
\text { therapy: No vs } \\
\text { Yes }\end{array}$ & $\begin{array}{l}1.481 \\
(0.591-3.741)\end{array}$ & 0.401 & & \\
\hline
\end{tabular}

Notes: $\mathrm{AEH}=$ atypical endometrial hyperplasia, $\mathrm{EC}=$ endometrial carcinoma.

Table 4 Pregnancy related factors

\begin{tabular}{|c|c|c|c|c|}
\hline $\begin{array}{l}\text { Predictors to } \\
\text { pregnancy }\end{array}$ & $\begin{array}{l}\text { Univariate analysis } \\
\text { HR }(95 \% \text { CI })\end{array}$ & P-value & $\begin{array}{l}\text { Multivariate } \\
\text { analysis HR }(95 \% \\
\text { CI) }\end{array}$ & $\mathrm{P}$-value \\
\hline $\begin{array}{l}\text { Age: }<35 \text { years } \\
\text { vs }>35 \text { years }\end{array}$ & $\begin{array}{l}4.373 \\
(1.427-13.406)\end{array}$ & 0.006 & $\begin{array}{l}5.246 \\
(1.638-16.802)\end{array}$ & 0.005 \\
\hline $\begin{array}{l}\text { Comorbidity: Yes } \\
\text { vs No }\end{array}$ & $\begin{array}{l}1.194 \\
(0.513-2.782)\end{array}$ & 0.681 & & \\
\hline $\begin{array}{l}\text { Obesity: No vs } \\
\text { Yes }\end{array}$ & $\begin{array}{l}2.688 \\
(1.184-6.103)\end{array}$ & 0.016 & $\begin{array}{l}2.598 \\
(1.103-6.119)\end{array}$ & 0.029 \\
\hline $\mathrm{AEH}$ vs $\mathrm{EC}$ & $0.733(0.325-1.656)$ & 0.455 & & \\
\hline
\end{tabular}


Loss-weight:

$[?] 3 \%$ vs $<3 \%$

IVF-ET: Yes vs

No

1.676

(0.800-3.512)

2.231

(1.031-4.824)
0.171

0.039

2.098

0.069

(0.919-4.790)

Notes: $\mathrm{AEH}=$ atypical endometrial hyperplasia, $\mathrm{EC}=$ endometrial carcinoma, $\mathrm{ART}=$ assisted reproductive technology, IVF-ET=in vitro fertilization and embryo transfer.
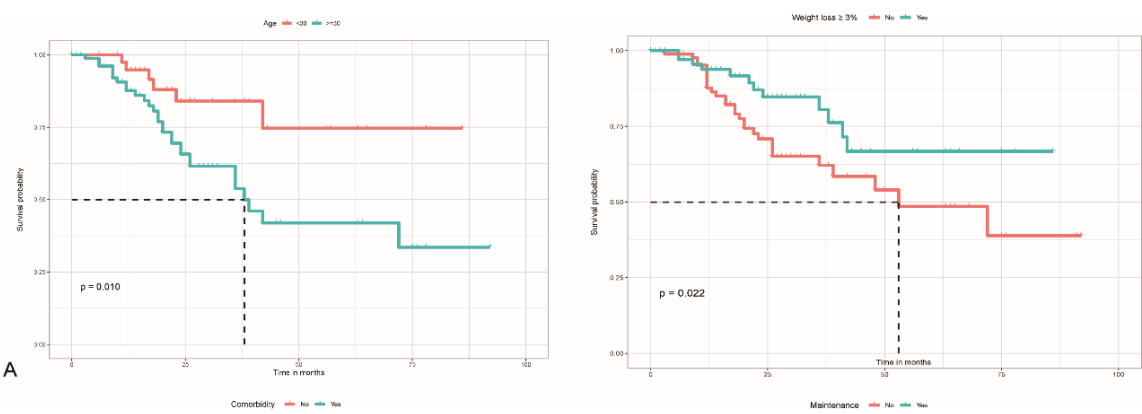

Figure 1 Kaplan Meier disease-free survival analysis for Age(A), and Weight loss (B) 